\title{
High-resolution Brillouin fiber sensing using random phase coding of the pump and probe waves
}

\author{
Yair Antman ${ }^{\mathrm{a}}$, Nikolay Primerov ${ }^{\mathrm{b}}$, Luc Thévenaz , and Avi Zadok ${ }^{\text {*a }}$ \\ ${ }^{a}$ Faculty of Engineering, Bar-Ilan University, Ramat-Gan 52900 Israel; \\ ${ }^{\mathrm{b}}$ Ecole Polytechnique Fédérale de Lausanne, Institute of Electrical Engineering, \\ SCI-STI-LT Station 11, 1015 Lausanne, Switzerland;
}

\begin{abstract}
Distributed temperature measurements with $1.2 \mathrm{~cm}$ resolution based on stimulated Brillouin scattering (SBS) in standard fibers are reported. High resolution is achieved by phase-coding both pump and probe waves with a high-rate, pseudorandom binary phase code. The SBS interaction is effectively confined to narrow correlation peaks. The separation between adjacent peaks, signifying the unambiguous measurement range, scales with the length of the modulation code and can therefore be made arbitrarily long. Measurements were performed over 40 meters of fiber, or 3300 resolution points. The technique is applicable to distributed measurements of birefringence and Brillouin frequency shift over polarization maintaining fibers.
\end{abstract}

Keywords: Stimulated Brillouin scattering, fiber-optics sensors, optical signal processing, distributed temperature measurements.

\section{INTRODUCTION}

Commercially available Brillouin optical time domain analysis (B-OTDA) instruments provide measurement ranges of tens of $\mathrm{km}$, with a spatial resolution of tens of $\mathrm{cm}$. In many applications, however, such as structural monitoring in the aerospace industry, a higher resolution is necessary. Standard B-OTDA techniques, which rely on the transmission of pulsed pump or probe waves, are restricted to meter-scale resolution: the use of pulses shorter than the acoustic damping time of $\tau \sim 12$ ns both weakens the SBS interaction and broadens its spectrum ${ }^{1-4}$. In recent years, more elaborate BOTDA methods had employed multiple pulse patterns and multiple scans to provide an order-of-magnitude higher resolution, at the cost of added complexity ${ }^{5-8}$.

Alternatively, Brillouin optical correlation domain analysis (B-OCDA) methods rely on synchronized frequency modulation of both pump and probe waves to effectively confine the interaction to narrow correlation peaks only ${ }^{9-10}$. BOCDA demonstrations had reached mm-scale resolution. However, in most implementations the frequencies of pump and probe are modulated by a simple sine wave, which provides separation of only several hundred resolution points between adjacent peaks and sets a tight tradeoff between range and resolution. The technique was recently extended to dual-tone frequency modulation ${ }^{11}$, resulting in a resolution of $27 \mathrm{~cm}$ over $1.5 \mathrm{~km}$. Nevertheless, Brillouin-based distributed sensing, with $\mathrm{cm}$-scale resolution and a large number of resolution points, remains challenging.

In this work, we report distributed Brillouin-based temperature measurements with a $1 \mathrm{~cm}$ resolution and a $0.5{ }^{\circ} \mathrm{C}$ sensitivity, over $40 \mathrm{~m}$ of fiber. High resolution is obtained through synchronized phase-coding of both pump and probe by a high-rate, binary pseudo-random bit sequence (PRBS), as described below. The range of unambiguous measurement scales with the length of the PRBS code, and can therefore be made arbitrarily long. This technique had recently been employed in the generation of localized dynamic acoustic gratings in polarization maintaining (PM) fibers, implementing variable delay setups ${ }^{12}$ and tunable microwave-photonic filters ${ }^{13}$.

\footnotetext{
*Avinoam.Zadok@biu.ac.il Tel.: +972-3-5318882; Fax: +972-3-7384051.
} 


\section{PRINCIPLE OF OPERATION}

The underlying principle of localizing the SBS interaction through coding of pump and probe waves is described with mathematical rigor elsewhere in another context ${ }^{12}$. A more intuitive explanation will be described here. Both Brillouin pump and signal waves are jointly phase-modulated by a common binary pseudo random bit sequence (PRBS), whose symbol duration $T$ is much shorter than the acoustic damping time $\tau^{12}$. The modulation phase within each symbol assumes a value of either 0 or $\pi$, with equal probabilities. Suppose the phase modulation is synchronized so that the two waves are of equal phases at their respective entry points. The instantaneous driving force for the SBS acoustic field generation is proportional to the product of the pump wave envelope and the complex conjugate of the signal wave envelope $^{5,12}$.

As illustrated in figure 1, we may distinguish between the dynamics of the SBS-induced acoustic field in two different regions. In the vicinity of the fiber center the envelopes of the pump and signal are correlated, hence their phase difference is constant and the driving force for the acoustic field generation keeps a steady non-zero value. Consequently, the acoustic field is allowed to build up to its steady state value, and permits the interrogation of the local Brillouin shift. The width of the correlation peak is on the order of $\Delta z=\frac{1}{2} v_{g} T$, where $v_{g}$ is the group velocity of light in the fiber ${ }^{12}$. In all other locations, the driving force for the acoustic field is randomly alternating in sign on every symbol duration $T<<\tau$. The acoustic field magnitude thus averages out to a zero expectation value, and the SBS interaction outside the correlation peak is largely inhibited ${ }^{11}$. The separation between adjacent correlation peaks equals $\frac{1}{2} M v_{g} T$, where $M$ is the PRBS length. The unambiguous measurement range can be made arbitrarily long, while retaining the above high resolution $\Delta z^{12}$. PRBS generators readily provide sequences that are $2^{31}-1$ bits long, corresponding to more than 2 billion resolved points. The correlation peaks, except the zeroth-order one, can be scanned along a fiber under test through changing either $M$ or $T$.

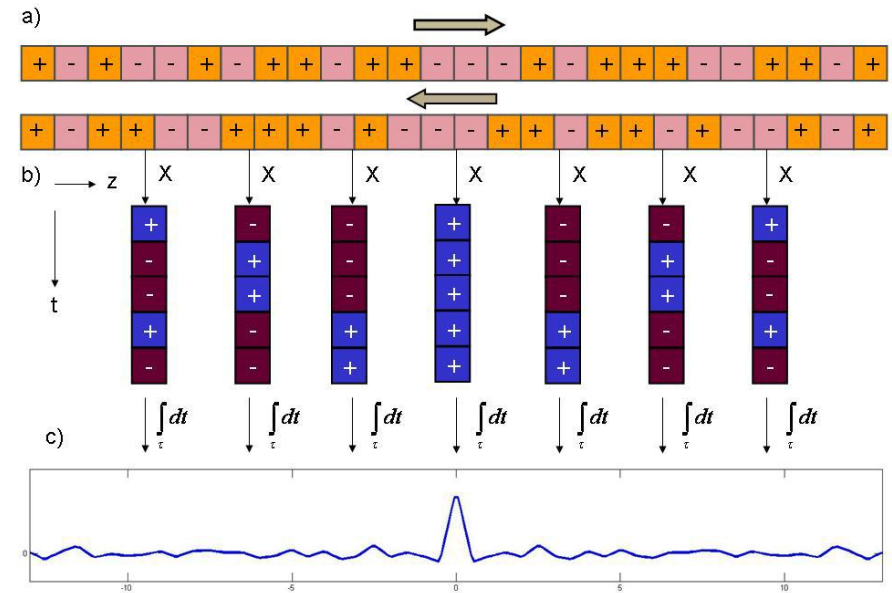

Fig. 1: a) Illustration of binary phase modulated SBS probe wave complex envelope (top row), and the SBS pump wave complex envelope (bottom row), co-modulated by the same binary phase sequence. b) A constant driving force for the acoustic field generation prevails at discrete peak locations only (centre), in which the two replicas of the modulation sequence are in correlation. Elsewhere, the driving force is oscillating. c) The magnitude of the resulting acoustic field, obtained by temporal integration over the driving force.

\section{EXPERIMENTAL SETUP AND RESULTS}

The measurement setup is shown in figure 2. Light from a distributed feedback (DFB) laser diode of frequency $\omega_{0}$ was used as the source of both pump and signal. The DFB output passed through an electro-optic phase modulator, driven by a PRBS generator. The modulation rate was on the order of $8 \mathrm{GHz}$, corresponding to a spatial resolution $\Delta z$ of $1.2 \mathrm{~cm}$, and the code length was $2^{15}-1=32,767$ symbols, representing the potential number of resolved points. The modulated 
wave was split into probe and pump paths. In the probe branch, a carrier-suppressed amplitude electro-optic modulator was used to generate two modulation sidebands that were detuned from $\omega_{0}$ by $\pm \Omega$. The probe wave was amplified and launched into one end of a fiber under test. Light in the pump branch was amplitude-modulated by a low-frequency sine wave, amplified to $500 \mathrm{~mW}$, and launched into the opposite end of the fiber under test. A delay imbalance was placed at the signal input end of the fiber, so that the seventh-order correlation peak of the modulation sequence overlapped the section of fiber under test. Specific fiber locations were accessed via slight adjustments of the PRBS clock rate: variation by $1 \mathrm{MHz}$ corresponded to an offset of the correlation peak by $34 \mathrm{~cm}$. A polarization scrambler was used in the pump arm, in order to avoid potential polarization-related fading. The output signal was filtered by a fiber-Bragg grating which retained only the sideband of interest at $\omega_{0}-\Omega$, then detected by a photo-diode. The detected current was analyzed by the lock-in-amplifier, tuned to the low modulation frequency of the pump wave.

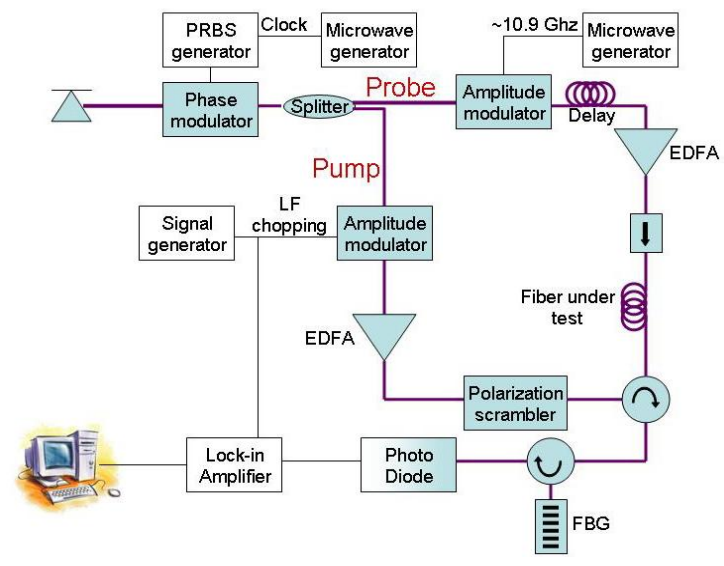

Fig. 2: Experimental setup for high-resolution SBS distributed temperature measurements using phase coding of pump and probe waves.
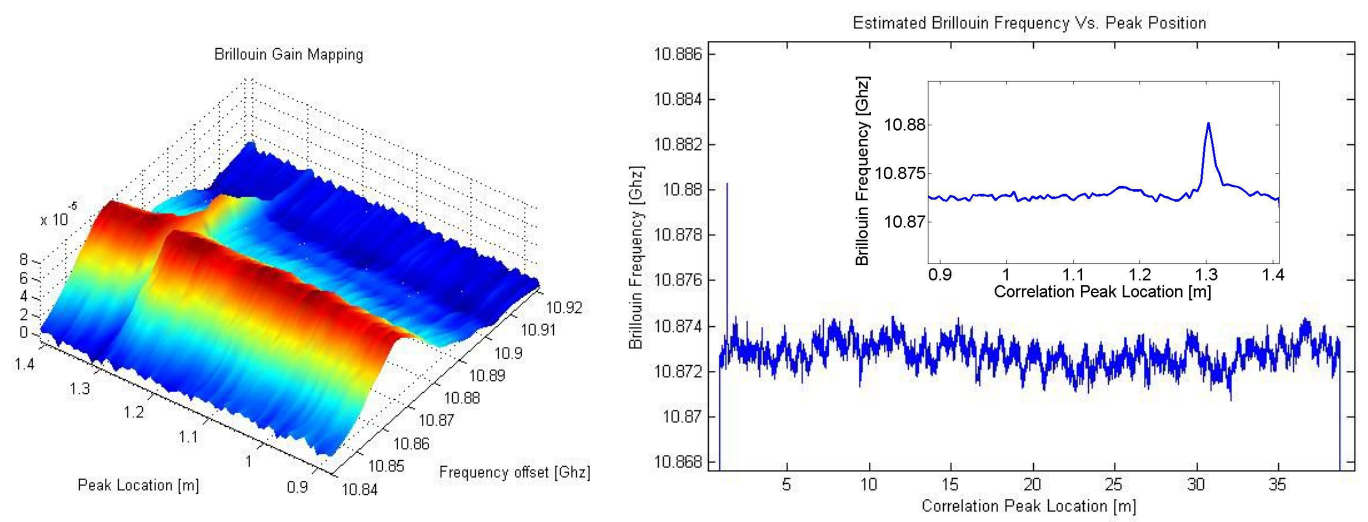

Fig. 3 Left - Measured Brillouin gain as a function of position and frequency shift between pump and probe. The gain map is magnified in the vicinity of a $1 \mathrm{~cm}$-long heated section, along a $40 \mathrm{~m}$-long standard fiber under test. Right measured Brillouin frequency shift as a function of position along the $40 \mathrm{~m}$ fiber. A magnification of the area surrounding the heated region is shown in the inset. 
Figure 3 shows an example of the measured Brillouin frequency shift as a function of position for a $40 \mathrm{~m}$ long standard fiber under test, along which a $1 \mathrm{~cm}$ long section had been locally heated. The measurements are able to resolve and locate the heated section. The range of the measurement was only restricted by the rate of addressing laboratory instrumentation, and it is scalable to km lengths.

The technique offers all the functionalities of B-OCDA, with the advantage of an arbitrary number of resolved points, and no inconvenience related to the frequency dithering of the laser source. As in B-OCDA, low-bandwidth detection is sufficient. The performance is therefore not limited by the response of the source to frequency modulation, and is entirely given by the PRBS characteristics. The spatial resolution, governed by the PRBS clock rate, is entirely decorrelated from the number of free resolved points, which is given by the PRBS code length.

\section{ACKNOWLEDGEMENTS}

This work was realized in the framework of the COST Action TD1001. The authors acknowledge support from the Swiss National Office for Education and Research through Project COST C06.0015 and SNF 20020-121860, the Israeli Science Foundation (ISF) and the KAMIN program of the Chief Scientist Office, Israeli Ministry of Industry, Trade and Labor (MOITAL).

\section{REFERENCES}

[1] Horiguchi, T., Kurashima, T., and Tateda, M., "A technique to measure distributed strain in optical fibers," IEEE Photon. Technol. Lett. 2, 352-354 (1990).

[2] Bao, X., Webb, D. J., and Jackson, D. A., "22-km distributed temperature sensor using Brillouin gain in an optical fiber," Opt. Lett. 18, 552-554 (1993).

[3] Niklès, M., Thévenaz, L., and Robert, P. A., "Simple distributed fiber sensor based on Brillouin gain spectrum analysis," Opt. Lett. 21, 758-760 (1996)

[4] Fellay, A., Thevenaz, L., Facchini, M., Nikles M., and Robert, P., "Distributing sensing using stimulated Brillouin scattering: Toward ultimate resolution," in Proceedings of the Optical Fiber Sensors Conference (OFS-12), 324-327 (1997).

[5] Foaleng, S. M., Tur, M., Beugnot, J. C., and Thevenaz, L., "High Spatial and Spectral Resolution Long-Range Sensing Using Brillouin Echoes," Journal of Lightwave Technology 28, 2993-3003 (2010).

[6] Bao, X., and Chen, L. A., "Recent progress in Brillouin scattering based fiber sensors," Sensors 11, 4152-4187 (2011).

[7] Thévenaz, L., "Brillouin distributed time-domain sensing in optical fibers: state of the art and perspectives," Frontiers of Optoelectronics in China 3, 13-21 (2010).

[8] Chin, S., Primerov, N., and Thevenaz, L., "Sub-Centimeter Spatial Resolution in Distributed Fiber Sensing Based on Dynamic Brillouin Grating in Optical Fibers," IEEE Sensors Journal 12, 189-194 (2012).

[9] Hotate, K., and Hasegawa, T., "Measurement of Brillouin gain spectrum distribution along an optical fiber using a correlation-based technique - proposal, experiment and simulation," IEICE Trans. Electron. E83-C, 405-412 (2000).

[10]Hotate, K., and Tanaka, M., "Distributed fiber Brillouin strain Sensing with 1-cm spatial resolution by correlation-based continuous-wave technique," IEEE Photon. Technol. Lett. 14, 179-181 (2002).

[11] Mizuno Y., He, Z. Y., and Hotate, K., "Measurement range enlargement in Brillouin optical correlation-domain reflectometry based on double-modulation scheme," Opt. Express 18, 5926-5933 (2010).

[12] Antman, Y., Primerov, N., Sancho, J., Thevenaz, L., and Zadok, A., "Localized and stationary dynamic gratings via stimulated Brillouin scattering with phase modulated pumps," Opt. Express 20, 7807-7821 (2012).

[13] Sancho, J., Primerov, N., Chin, S., Antman, Y., Zadok, A., Sales, S., and Thévenaz, L., "Tunable and reconfigurable multi-tap microwave photonic filter based on dynamic Brillouin gratings in fibers," Opt. Express 20, 6157-6162 (2012). 